\title{
EXTENSION OF SOLUTIONS FOR MONGE-AMPÈRE EQUATIONS OF HYPERBOLIC TYPE
}

\author{
MIKIO TSUJI \\ Department of Mathematics, Kyoto Sangyo University \\ Kita-ku, Kyoto 603, Japan
}

1. Introduction. In this talk we consider the Cauchy problem for real MongeAmpère equations of hyperbolic type. If the equations are not degenerate, it is well known that the Cauchy problem for (2.1) below has locally a smooth solution (see, for example, [1]-[5], [8], [9]). Our interest is in the global behaviour of solutions. But we cannot expect that it admits a smooth solution in the large, especially in hyperbolic case. This means that, if we extend the smooth solution, singularities may appear. The aim of this talk is to construct the singularities of the solutions in the case where the equations are hyperbolic. For our aim, we have to represent the solutions explicitly. To do so, we apply the characteristic method developed principally by G. Darboux and E. Goursat ([2]-[4]). As it seems to us that the method is not familiar today, we will briefly present it in $\S 2$. But this method depends on the dimension of the space being two. Therefore we will discuss, in $\S 3$, the principal difference between the equation in the two-dimensional space and equations of Monge-Ampère type in higher dimensions. By doing so, we can understand the geometric structure of the equations. In $\S 4$, we will construct the singularities of solutions of the equations, using the results of [15] and [16]. This is the principal part of this note. Though there are a lot of beautiful global theories for elliptic Monge-Ampère equations, it seems to us that we have almost nothing on global treatment of hyperbolic Monge-Ampère equations. Therefore we think that, though we assume in this note rather strong conditions, this is one step to developing a global theory. In this note we will give only a sketch of our approach to the above problem. The detailed paper will be published elsewhere.

The author thanks B. Gaveau, A. Kaneko, J. Vaillant, D. Gourdin and T. Morimoto for valuable suggestions and many useful conversations.

2. Characteristic method. For an unknown function $z=z(x, y)$ defined for $(x, y) \in$

1991 Mathematics Subject Classification: Primary 35L70; Secondary 35L25, 58C27, 76L05. The detailed version of this paper will appear elsewhere. 
$\mathbb{R}^{2}$, we denote $p=\partial z / \partial x, q=\partial z / \partial y, r=\partial^{2} z / \partial x^{2}, s=\partial^{2} z / \partial x \partial y$, and $t=\partial^{2} z / \partial y^{2}$. Monge-Ampère equations are written as

$$
F(x, y, z, p, q, r, s, t)=A r+B s+C t+D\left(r t-s^{2}\right)-E=0
$$

where $A, B, C, D$ and $E$ are real smooth functions of $(x, y, z, p, q)$. In this note, a "smooth" function means that it is of class $C^{\infty}$. Let

$$
C: \quad(x, y, z, p, q)=(x(\alpha), y(\alpha), z(\alpha), p(\alpha), q(\alpha)), \quad \alpha \in \mathbb{R}^{1},
$$

be a smooth curve in $\mathbb{R}^{5}$, and suppose that it satisfies the following "strip condition":

$$
\frac{d z}{d \alpha}(\alpha)=p(\alpha) \frac{d x}{d \alpha}(\alpha)+q(\alpha) \frac{d y}{d \alpha}(\alpha)
$$

As a "characteristic strip" means that one cannnot determine the value of the second derivatives of a solution along the strip $C$, we have the following

Definition 2.1. A curve $C$ in the space with coordinates $(x, y, z, p, q)$ is a characteristic strip if it satisfies (2.2) and

$$
\operatorname{det}\left[\begin{array}{ccc}
F_{r} & F_{s} & F_{t} \\
\dot{x} & \dot{y} & \dot{0} \\
0 & \dot{x} & \dot{y}
\end{array}\right]=F_{t} \dot{x}^{2}-F_{s} \dot{x} \dot{y}+F_{r} \dot{y}^{2}=0
$$

where $F_{t}=\partial F / \partial t, F_{s}=\partial F / \partial s, F_{r}=\partial F / \partial r, \dot{x}=d x / d \alpha$ and $\dot{y}=d y / d \alpha$.

Denote the discriminant of (2.3) by $\Delta$. Then $\Delta=F_{s}^{2}-4 F_{r} F_{t}=B^{2}-4(A C+D E)$. If $\Delta<0$, the equation (2.1) is called elliptic. If $\Delta>0$, the equation (2.1) is hyperbolic. In this note, we will treat the equations of hyperbolic type. More precisely, we assume $\Delta \geq 0$ and also $D \neq 0$. Let $\lambda_{1}$ and $\lambda_{2}$ be the solutions of $\lambda^{2}+B \lambda+(A C+D E)=0$. Then the characteristic strip satisfies the following equations:

$$
\left\{\begin{array}{l}
d z-p d x-q d y=0 \\
D d p+C d x+\lambda_{1} d y=0 \\
D d q+\lambda_{2} d x+A d y=0
\end{array}\right.
$$

or

$$
\left\{\begin{array}{l}
d z-p d x-q d y=0 \\
D d p+C d x+\lambda_{2} d y=0 \\
D d q+\lambda_{1} d x+A d y=0 .
\end{array}\right.
$$

Definition 2.2. A function $V=V(x, y, z, p, q)$ is called a first integral of (2.4) (or (2.5)) if it is constant on any solution of (2.4) (or of (2.5) respectively).

Assume that the system (2.4) or (2.5) has at least two independent first integrals. We denote them by $u$ and $v$. For any function $g$ of two variables whose gradient does not vanish, $g(u, v)=0$ is called an intermediate integral of (2.1). Let $C_{0}$ be an initial strip defined in $\mathbb{R}^{5}=\{(x, y, z, p, q)\}$. If the strip $C_{0}$ is not characteristic, we can find an intermediate integral $g(u, v)$ which vanishes on $C_{0}$. Here we put $f(x, y, z, p, q)=$ $g(u, v)$. The Cauchy problem for (2.1) with the initial condition $C_{0}$ is to look for a solution $z=z(x, y)$ of (2.1) which contains the strip $C_{0}$, i.e., the two-dimensional surface $\left\{(x, y, z(x), \partial z / \partial x(x, y), \partial z / \partial y(x, y)\}\right.$ in $\mathbb{R}^{5}$ contains the strip $C_{0}$. Then G. Darboux [2] and E. Goursat [3] obtained the following 
THEOREM 2.3. Assume that the initial strip $C_{0}$ is not characteristic. Then a solution of the Cauchy problem for (2.1) with the initial condition $C_{0}$ is locally obtained as a solution of $f(x, y, z, \partial z / \partial x, \partial z / \partial y)=0$ with the same initial condition.

3. Representation of equations by differential forms. In this section we will consider the equation (2.1) from the point of view of differential forms. Concerning this subject, we have been stimulated by [10] and [11]. Assume in this section $D \neq 0$ and $\Delta \neq 0$, though we may treat the degenerate case in other sections. Let us denote $\omega_{0}=$ $d z-d x-q d y, \omega_{1}=D d p+C d x+\lambda_{1} d y$ and $\omega_{2}=D d q+\lambda_{2} d x+A d y$. Take an exterior product of $\omega_{1}$ and $\omega_{2}$ and substitute into their product the contact relations $\omega_{0}=0$, $d p=r d x+s d y$ and $d q=s d x+r d y$. Then we get

$$
\omega_{1} \wedge \omega_{2}=D\left\{A r+B s+C t+D\left(r t-s^{2}\right)-E\right\} d x \wedge d y .
$$

The important property of (2.1) is that every non-degenerate equation of type $(2.1)$ is decomposed as a product of one-forms as (3.1). This is true even if $D \equiv 0$ and $\Delta \equiv 0$. A necessary and sufficient condition for $u=u(x, y, z, p, q)$ to be a first integral of $(2.4)$ is

$$
d u \equiv 0 \bmod \left\{\omega_{0}, \omega_{1}, \omega_{2}\right\} .
$$

As $D \neq 0$ by the assumption, we can substitute the relations $\omega_{0}=\omega_{1}=\omega_{2}=0$ into $D d u=0$. Then we have

$$
D d u=\left\{D \frac{\partial u}{\partial x}+p D \frac{\partial u}{\partial z}-C \frac{\partial u}{\partial p}-\lambda_{2} \frac{\partial u}{\partial q}\right\} d x+\left\{D \frac{\partial u}{\partial y}+q D \frac{\partial u}{\partial z}-\lambda_{1} \frac{\partial u}{\partial p}-A \frac{\partial u}{\partial q}\right\} d y=0 .
$$

Therefore a necessary and sufficient condition for a function $u=u(x, y, z, p, q)$ to be a first integral of (2.4) is that it satisfies the following system of linear partial differential equations:

$$
\left\{\begin{array}{l}
L_{1} u \equiv D \frac{\partial u}{\partial x}+p D \frac{\partial u}{\partial z}-C \frac{\partial u}{\partial p}-\lambda_{2} \frac{\partial u}{\partial q}=0 \\
L_{2} u \equiv D \frac{\partial u}{\partial y}+q D \frac{\partial u}{\partial z}-\lambda_{1} \frac{\partial u}{\partial p}-A \frac{\partial u}{\partial q}=0
\end{array}\right.
$$

Here we can write $d u$ and $d v$ as

$$
d u=c_{10} \omega_{0}+c_{11} \omega_{1}+c_{12} \omega_{2} \quad \text { and } \quad d v=c_{20} \omega_{0}+c_{21} \omega_{1}+c_{22} \omega_{2} .
$$

Suppose that $u$ and $v$ are independent first integrals of (2.4). Then we prove

$$
\operatorname{det}\left(\begin{array}{ll}
c_{11} & c_{12} \\
c_{21} & c_{22}
\end{array}\right) \neq 0 .
$$

Using (3.3) and the contact relations $\left\{\omega_{0}=0, d p=r d x+s d y, d q=s d x+r d y\right\}$, we get easily

$$
d u \wedge d v=\operatorname{det}\left(c_{i j}\right) \omega_{1} \wedge \omega_{2}=\operatorname{det}\left(c_{i j}\right) D\left\{A r+B s+C t+D\left(r t-s^{2}\right)-E\right\} d x \wedge d y .
$$

This representation means that, as $d u \wedge d v=0$ on a surface $g(u, v)=0$, a smooth solution of $f(x, y, z, \partial z / \partial x, \partial z / \partial y)=0$ satisfies the equation (2.1). Hence we can immediately get Theorem 2.3. 
In a space whose dimension is greater than two, the decomposition as above is not possible in general. For example, consider the following equation in $\mathbb{R}^{3}$ :

$$
\operatorname{det}\left(\frac{\partial^{2} z}{\partial x_{i} \partial x_{j}}\right)_{1 \leq i, j \leq 3}=f\left(x, z, z^{\prime}(x)\right)
$$

If $f(x, z, p)$ is not zero, we cannot get a decomposition of (3.5) into a product of oneforms as (3.1). Therefore we see that the theory of Darboux and Goursat explained in $\S 2$ depends essentially on the space dimension being two. But, if an equation of second order is decomposed into a product of one-forms, we can develop a theory similar to $\S 2$.

Let us write $x=\left(x_{1}, \ldots, x_{n}\right) \in \mathbb{R}^{n}$ and $z=z(x)$ be an unknown function. Denote $z^{\prime}(x)=(\operatorname{grad} z)(x)=\left(p_{1}, \ldots, p_{n}\right)=p$ and $z^{\prime \prime}(x)=\left\{\partial^{2} z / \partial x_{i} \partial x_{j}: i, j=1, \ldots, n\right\}=$ $\left\{s_{i j} ; i, j=1, \ldots, n\right\}=s$. We consider an equation

$$
F\left(x, z, z^{\prime}(x), z^{\prime \prime}(x)\right)=F(x, z, p, s)=0 .
$$

Let $\omega_{1}, \ldots, \omega_{n}$ be independent one-forms defined on $\mathbb{R}^{2 n+1}=\left\{(x, z, p): x, p \in \mathbb{R}^{n}, z \in\right.$ $\left.\mathbb{R}^{1}\right\}$. We take the product $\omega_{1} \wedge \ldots \wedge \omega_{n}$ and substitute there the following contact relations:

$$
\omega_{0} \equiv d z-\sum_{i=1}^{n} p_{i} d x_{i}=0, \quad d p_{i}-\sum_{j=1}^{n} s_{i j} d x_{j}=0 \quad(i=1, \ldots, n) \quad \text { and } \quad s_{i j}=s_{j i} .
$$

Our assumption is that the equation (3.6) is obtained by

$$
\omega_{1} \wedge \ldots \wedge \omega_{n}=F(x, z, p, s) d x_{1} \wedge \ldots \wedge d x_{n} .
$$

Definition 3.1. A function $u=u(x, z, p)$ is a first integral of the family of one-forms $\left\{\omega_{0}, \omega_{1}, \ldots, \omega_{n}\right\}$ if and only if $d u \equiv 0 \bmod \left\{\omega_{0}, \omega_{1}, \ldots, \omega_{n}\right\}$.

Let us put $A^{0}=\left(x^{0}, z^{0}, p^{0}\right) \in \mathbb{R}^{2 n+1}, \vec{r}=\left(a_{1}, \ldots, a_{n}, c, b_{1}, \ldots, b_{n}\right)$ and

$$
L=\sum_{i=1}^{n} a_{i} \frac{\partial}{\partial x_{i}}+c \frac{\partial}{\partial z}+\sum_{i=1}^{n} b_{i} \frac{\partial}{\partial p_{i}}
$$

Definition 3.2. A direction $\vec{r}$ is called a characteristic direction at $A^{0}$ if $\left\langle\omega_{i}, L\right\rangle=0$ at $A^{0}$ for any $i=0,1,2, \ldots, n$.

Definition 3.3. A smooth manifold $M$ is not characteristic with respect to $\left\{\omega_{0}, \ldots\right.$ $\left.\ldots, \omega_{n}\right\}$ if and only if the tangent space $T_{A}(M)$ at any point $A \in M$ does not contain any characteristic direction.

Let $C_{0}$ be an $(n-1)$-dimensional smooth submanifold in $\mathbb{R}^{2 n+1}=\{(x, z, p): x, p \in$ $\left.\mathbb{R}^{n}, z \in \mathbb{R}^{1}\right\}$. We assume that $C_{0}$ satisfies the strip condition, i.e.,

$$
d z-p_{1} d x_{1}-\ldots-p_{n} d x_{n}=0 \quad \text { on } C_{0} .
$$

The Cauchy problem for (3.6) with initial data $C_{0}$ is to look for a solution $z=$ $z(x)$ such that the surface $\left\{\left(x, z(x), z^{\prime}(x)\right) ; x \in \mathbb{R}^{n}\right\}$ contains $C_{0}$. Here we assume the conditions:

(H1) There exist $n$ independent first integrals of $\left\{\omega_{0}, \omega_{1}, \ldots, \omega_{n}\right\}$. We write them as $u_{1}(x, z, p), u_{2}(x, z, p), \ldots, u_{n}(x, z, p)$.

(H2) The initial manifold $C_{0}$ satisfies the strip condition (3.9) and is not characteristic with respect to $\left\{\omega_{0}, \omega_{1}, \ldots, \omega_{n}\right\}$. 
(H3) $\omega_{i}=d p_{i}+\sum_{j=1}^{n} a_{i j} d x_{j}$ for $i=1, \ldots, n$, where $a_{i j}$ are smooth functions of $(x, z, p)$ and the $n \times n$ matrix $\left[a_{i j}\right]$ is not symmetric.

Note that the assumption (H3) corresponds to the conditions $D=1$ and $\Delta \neq 0$ for (2.1). Though we can remove them in some cases, we assume them to make our discussion simple. Let $u=u(x, z, p)$ be a first integral in the sense of Definition 3.1. We substitute the contact relations (3.7) into $d u=0$. Then we have

$$
d u=\sum_{i=1}^{n} \frac{\partial u}{\partial x_{i}} d x_{i}+\frac{\partial u}{\partial z} d z+\sum_{i=1}^{n} \frac{\partial u}{\partial p_{i}} d p_{i}=\sum_{i=1}^{n}\left\{\frac{\partial u}{\partial x_{i}}+p_{i} \frac{\partial u}{\partial z}-\sum_{j=1}^{n} a_{j i} \frac{\partial u}{\partial p_{j}}\right\} d x_{i}=0 .
$$

Therefore we have

LEMMA 3.4. A necessary and sufficient condition for $u=u(x, z, p)$ to be a first integral in the sense of Definition 3.1 is that $u(x, z, p)$ satisfies the following system of linear partial differential equations:

$$
L_{i} u=\frac{\partial u}{\partial x_{i}}+p_{i} \frac{\partial u}{\partial z}-\sum_{j=1}^{n} a_{j i} \frac{\partial u}{\partial p_{j}}=0 \quad(i=1, \ldots, n) .
$$

Let $u_{i}(i=1, \ldots, n)$ be first integrals in the sense of Definition 3.1. Then $d u_{i}$ is represented as

$$
d u_{i}=c_{i 0} \omega_{0}+c_{i 1} \omega_{1}+\ldots+c_{i n} \omega_{n} \quad(i=1, \ldots, n) .
$$

Assume the conditions (H1) and (H3). Then we can prove $\operatorname{det}\left[c_{i j}\right]_{1 \leq i, j \leq n} \neq 0$.

Now we consider the Cauchy problem for the equation (3.6). Let us write the initial submanifold $C_{0}$ as follows:

$$
\begin{array}{r}
C_{0}: \quad x_{i}=x_{i}\left(\alpha_{1}, \ldots, \alpha_{n-1}\right), \quad z=z\left(\alpha_{1}, \ldots, \alpha_{n-1}\right), \quad p_{i}=p_{i}\left(\alpha_{1}, \ldots, \alpha_{n-1}\right) \\
(i=1, \ldots, n) .
\end{array}
$$

Assume the conditions $(\mathrm{H} 1)$ and $(\mathrm{H} 2)$. Then we can find a function $g=g\left(u_{1}, \ldots, u_{n}\right)$ with the following two properties: 1) $g\left(u_{1}, \ldots, u_{n}\right)$ vanishes on $C_{0}$, and 2$)\left(\partial g / \partial u_{1}, \ldots\right.$ $\left.\ldots, \partial g / \partial u_{n}\right) \neq(0, \ldots, 0)$.

Definition 3.5. When $\left(\partial g / \partial u_{1}, \ldots, \partial g / \partial u_{n}\right) \neq(0, \ldots, 0)$, we call the equation $g\left(u_{1}, \ldots, u_{n}\right)=0$ an intermediate integral of the equation (3.6).

Here we put $f(x, z, p)=g\left(u_{1}, \ldots, u_{n}\right)$, and consider the Cauchy problem

$$
f(x, z, \partial z / \partial x)=0, \quad(z, \partial z / \partial x)=(z(\alpha), p(\alpha)) \quad \text { for } x=x(\alpha)
$$

where $\alpha=\left(\alpha_{1}, \ldots, \alpha_{n-1}\right)$ and $\partial z / \partial x=\left(\partial z / \partial x_{1}, \ldots, \partial z / \partial x_{n}\right)$. As $C_{0}$ is not characteristic, the Cauchy problem (3.12) has locally a smooth solution. On the solution surface, using (3.7) and (3.11), we get

$$
d u_{1} \wedge \ldots \wedge d u_{n}=\operatorname{det}\left(c_{i j}\right) \omega_{1} \wedge \ldots \wedge \omega_{n}=\operatorname{det}\left(c_{i j}\right) F(x, z, p, s) d x_{1} \wedge \ldots \wedge d x_{n} .
$$

As $d u_{1} \wedge \ldots \wedge d u_{n}=0$ on the surface $g\left(u_{1}, \ldots, u_{n}\right)=0$, the smooth solution of $f\left(x, z, z^{\prime}(x)\right)$ $=0$ satisfies the equation (3.6). Summing up the above discussion, we have 
TheOrem 3.6. Assume the conditions (H1), (H2) and (H3). Then a solution of the Cauchy problem for (3.6) with the initial condition $C_{0}$ is locally obtained as a solution of the equation $f\left(x, z, z^{\prime}(x)\right)=0$ with the same initial condition.

ExAmPle. Let

$$
\omega_{i}=d p_{i}+\sum_{j=1}^{n} a_{i j} d x_{j} \quad(i=1, \ldots, n)
$$

and assume that the coefficients $\left\{a_{i j}\right\}$ are all constant. Then the Monge-Ampère equation obtained from $\left\{\omega_{0}, \omega_{1}, \ldots, \omega_{n}\right\}$ via (3.8) satisfies the condition (H.1). In this case, the equation is written as

$$
F\left(x, z, z^{\prime}, z^{\prime \prime}\right)=\operatorname{det}\left(\frac{\partial^{2} z}{\partial x_{i} \partial x_{j}}+a_{i j}\right)_{1 \leq i, j \leq n}=0
$$

and first integrals $\left\{u_{i}\right\}_{i=1, \ldots, n}$ are given by $u_{i}=p_{i}+\sum_{j=1}^{n} a_{i j} x_{j}(i=1, \ldots, n)$.

4. The Cauchy problem for Monge-Ampère equations (2.1). Let $C_{0}$ be a smooth strip defined in $\mathbb{R}^{5}=\{(x, y, z, p, q)\}$ as follows:

$$
C_{0}: \quad x=x(\alpha), y=y(\alpha), z=z(\alpha), p=p(\alpha), q=q(\alpha), \quad \alpha \in \mathbb{R}^{1} .
$$

We consider the Cauchy problem for the Monge-Ampère equation (2.1) with the initial condition $C_{0}$, i.e. look for a solution $z=z(x, y)$ which passes through the strip $C_{0}$ in the sense explained after Definition 2.2 in $\S 2$. We assume the conditions:

(A.1) The system of equations (2.4) (or (2.5)) has two independent first integrals; denote them by $u$ and $v$.

(A.2) The initial strip $C_{0}$ is not characteristic.

Denote by $u_{0}(\alpha)$ the value of $u$ restricted to $C_{0}$ and by $v_{0}(\alpha)$ the value of $v$ restricted to $C_{0}$, and $T=\left\{(u, v): u=u_{0}(\alpha), v=v_{0}(\alpha)\right.$ for any $\left.\alpha \in \mathbb{R}^{1}\right\}$. As we get $\left(u_{0}^{\prime}, v_{0}^{\prime}\right) \neq(0,0)$ by the condition (A.2), we can show the local existence of a function $g(u, v)$ satisfying $g\left(u_{0}(\alpha), v_{0}(\alpha)\right)=0$ and $(\operatorname{grad} g)\left(u_{0}(\alpha), v_{0}(\alpha)\right) \neq(0,0)$. But we would like to develop a global theory. Therefore $g(u, v)$ should be defined in the large with the above two properties. To obtain such a function $g(u, v)$ in the whole space, we assume the following condition:

(A.3) The curve $T$ is contained in a smooth curve which divides the whole plane $\mathbb{R}^{2}$ into two unbounded and connected regions.

Here we put $g(u, v)=f(x, y, z, p, q)$, and consider the Cauchy problem:

$$
\left\{\begin{array}{l}
f(x, y, z, \partial z / \partial x, \partial z / \partial y)=0, \\
(z(x(\alpha), y(\alpha))=z(\alpha),(\partial z / \partial x)(x(\alpha), y(\alpha))=p(\alpha),(\partial z / \partial y)(x(\alpha), y(\alpha))=q(\alpha) .
\end{array}\right.
$$

As we have explained in $\S 2$ and $\S 3$, a solution of the Cauchy problem $(2.1)-(4.1)$ is locally obtained from a solution of (4.2). Extending the solution of (4.2), we see the global behaviour of the solution of (2.1)-(4.1). Now we solve the Cauchy problem (4.2). 
The characteristic differential equations for (4.2) are written down as follows:

$$
\left\{\begin{array}{l}
\frac{d x}{d \beta}=\frac{\partial f}{\partial p}(x, y, z, p, q), \quad \frac{d y}{d \beta}=\frac{\partial f}{\partial q}(x, y, z, p, q), \\
\frac{d z}{d \beta}=p \frac{\partial f}{\partial p}(x, y, z, p, q)+q \frac{\partial f}{\partial q}(x, y, z, p, q), \\
\frac{d p}{d \beta}=-\frac{\partial f}{\partial x}(x, y, z, p, q)-p \frac{\partial f}{\partial z}(x, y, z, p, q), \\
\frac{d q}{d \beta}=-\frac{\partial f}{\partial y}(x, y, z, p, q)-q \frac{\partial f}{\partial z}(x, y, z, p, q), \\
x(0)=x(\alpha), y(0)=y(\alpha), z(0)=z(\alpha), p(0)=p(\alpha), q(0)=q(\alpha), \quad \alpha \in \mathbb{R}^{1} .
\end{array}\right.
$$

Writing the solutions of (4.3)-(4.4) as $x=x(\alpha, \beta), y=y(\alpha, \beta), z=z(\alpha, \beta), p=$ $p(\alpha, \beta), q=q(\alpha, \beta)$, we put $X=\{(x, y, z, p, q): x=x(\alpha, \beta), y=y(\alpha, \beta), z=z(\alpha, \beta)$, $p=p(\alpha, \beta), q=q(\alpha, \beta)\}$. As $\omega_{0}=d z-p d x-q d y=0$ on $X$, we see that $X$ is a two-dimensional Legendrian submanifold in $\mathbb{R}^{5}$. Let $\pi$ be the projection from $\mathbb{R}^{5}$ to $\mathbb{R}^{3}$ defined by $\pi(x, y, z, p, q)=(x, y, z)$ and $H$ be the smooth mapping from $\mathbb{R}^{2}$ to $\mathbb{R}^{2}$ defined by $H(\alpha, \beta)=(x(\alpha, \beta), y(\alpha, \beta))$. Denote $S=\pi(X)$.

Definition 4.1. For any set $S$ in $\mathbb{R}^{3}$, we call $X$ a Legendrian lift of $S$ if $\pi(X)=S$ and $X$ is a two-dimensional Legendrian submanifold.

Let us explain the reason for introducing the above notion. It is well known that the family of characteristic rays constitutes a Legendrian submanifold in the cotangent space. In a neighbourhood of $\pi(P)$ where $P$ is a regular point of the projection $\pi$, a smooth solution is obtained as the image of a Legendrian submanifold by $\pi$. Our aim is to show that every solution is obtained as the projection of a Legendrian submanifold into the base space, even if the solution may contain singularities. Our following discussion is just carrying out this procedure. For example, see Theorem 4.6 and the remark.

Theorem 2.3 means that $S$ is a smooth solution surface of (2.1)-(4.1) in a neighbourhood of a point where the mapping $H$ is regular. As $\omega_{0}=0$ on $X$, we have the following

Lemma 4.2. Suppose that $p(\alpha, \beta)$ and $q(\alpha, \beta)$ exist, then

$$
\operatorname{rank}\left(\begin{array}{ccc}
\frac{\partial x}{\partial \alpha} & \frac{\partial y}{\partial \alpha} & \frac{\partial z}{\partial \alpha} \\
\frac{\partial x}{\partial \beta} & \frac{\partial y}{\partial \beta} & \frac{\partial z}{\partial \beta}
\end{array}\right)=\operatorname{rank}\left(\begin{array}{ll}
\frac{\partial x}{\partial \alpha} & \frac{\partial y}{\partial \alpha} \\
\frac{\partial x}{\partial \beta} & \frac{\partial y}{\partial \beta}
\end{array}\right) \quad \text { for all }(\alpha, \beta) \in \mathbb{R}^{2} \text {. }
$$

This means that the surface $S$ is not regular at points where the mapping $H$ is singular, i.e. the Jacobian $D(x, y) / D(\alpha, \beta)$ vanishes. Moreover, if the Jacobian vanishes, a solution becomes multi-valued as a function of $(x, y)$. Here is just a transition point for our further discussions, and we have two ways to choose.

One way is to introduce a physical point of view. Then a solution must be singlevalued. For this purpose, we cut off some parts of the solution surface so that it could become a single-valued weak or generalized solution satisfying the entropy condition for equations of conservation laws or the semi-concavity condition for Hamilton-Jacobi 
equations. By this procedure, singularities may appear in the solution surface. If the space dimension is one, there are many works on construction of singularities of weak solutions. When the space dimension is two, see M. Tsuji [15]. S. Nakane [12, 13] considered the similar problem in the case of several space dimensions. But his situations can be regarded as the case where the space dimension is two, because he treated smooth mappings whose singularities are essentially "fold and cusp points" only. To consider the problem in several dimensions, one must treat smooth mappings whose singularities are not of Morin type. For this analysis, we need canonical forms of smooth mappings in a neighbourhood of a singular point. Recently, S. Izumiya [6] and S. Izumiya and G. T. Kossioris [7] gave canonical forms of smooth mappings, in the framework of "Legendrian unfoldings", which appear in the process of solving partial differential equations of first order.

Another way is to consider the above problem from the geometrical point of view. Then we must accept multi-valued solutions. As Monge-Ampère equations appear often in geometric problems, we should take here this second approach to the Cauchy problem (4.2). This means that, without cutting off some part of the solution surface, we should accept the whole solution surface $S$ and analyse it especially in a neighbourhood of a singular point of the mapping $H$. Let us represent the surface $S$ by $z=z(x, y)$. In [16] we have already studied the regularity of $S$ in neighbourhoods of singular points of $H$ under the following condition (A.4):

(A.4) The singularities of the mapping $H$ are fold and cusp points only.

Let us give some comments on this assumption. H. Whitney [17] proved that, if the space dimension is two, the subset of smooth mappings whose singularities are only fold and cusp points is open and dense in the space of smooth mappings from plane to plane. Therefore the assumption (A.4) is natural in generic sense.

From now on, we apply the results of $[16]$ to the above problem for (2.1). Let us write $\Sigma=\{(\alpha, \beta) ; D(x, y) / D(\alpha, \beta)=0\}$ and $H(\Sigma)=\Gamma$. As cusp points are isolated, we see that the curve $\Gamma$ is piecewise smooth and its singularities are cusps. Then we have the following

THEOREM 4.3 ([16]). 1) Let the equation (4.2) be a quasi-linear first order partial differential equation. Then the solution $z=z(x, y)$ is smooth except on the curve $\Gamma$, and it is $C^{0}$, but not $C^{1}$, in a neighbourhood of the curve $\Gamma$.

2) Suppose that the equation is not quasi-linear, i.e., of Hamilton-Jacobi type. Then the solution $z=z(x, y)$ is smooth except on the curve $\Gamma$, and it is $C^{1}$, but not $C^{2}$, in a neighbourhood of the curve $\Gamma$.

Remark. Let us explain the meaning of "Hamilton-Jacobi type". In [16], we have discussed the differences between Hamilton-Jacobi equations and equations of conservation laws, under the assumption that $f(x, y, z, p, q)$ is smooth. Our conclusion is that the most characteristic property of Hamilton-Jacobi equations is the global solvability of the Cauchy problem for (4.3). On the other hand, if $f=0$ is quasi-linear, the solution $p(\alpha, \beta)$ and $q(\alpha, \beta)$ tend to infinity when the Jacobian $D(x, y) / D(\alpha, \beta)$ vanishes. Therefore, in the above theorem, "Hamilton-Jacobi type" means the global solvability of the Cauchy problem for (4.3). 
If we assume the conditions (A.1), (A.2) and (A.3), we can easily prove the uniqueness of solution in the space of functions of class $C^{2}$. But Theorem 4.3 says that the solution $z=z(x, y)$ is not of class $C^{2}$ in a neighbourhood of $\Gamma$. Therefore we must discuss the uniqueness of extension of solution beyond the curve $\Gamma$ in $C^{1}$-space, or in $C^{0}$-space.

Let $A$ be on the curve $\Gamma$ and put $A=H(P)$. When $P$ is a cusp point of the mapping $H$, the canonical form of $H$ in a neighbourhood of $P$ is represented by a polynomial of degree 3 ([17]). Therefore the solution $z=z(x, y)$ becomes three-valued in a neighbourhood of the point $A$. When $P$ is not a cusp point, i.e., a fold point of $H$, we see by (A.4) that the curve $\Gamma$ is smooth in a neighbourhood of $A$. In fact, the curve $\Gamma$ is obtained as the envelope of a family of curves $\Phi=\left\{C_{\alpha}\right\}$ where $C_{\alpha}=\left\{(x, y) ; x=x(\alpha, \beta), y=y(\alpha, \beta), \beta \in \mathbb{R}^{1}\right\}$. The condition (A.4) shows that the family $\Phi=\left\{C_{\alpha}\right\}$ covers one side of the curve $\Gamma$. We denote this region by $D_{+}$and the region on the other side by $D_{-}$, and also write $z_{\Gamma}$ for the value of the solution $z=z(x, y)$ on the curve $\Gamma$. If the equation $f=0$ is of Hamilton-Jacobi type, we can determine the values of $\partial z / \partial x$ and $\partial z / \partial y$ on the curve $\Gamma$ ([16]) and denote them by $p_{\Gamma}$ and $q_{\Gamma}$ respectively. Here we recall the result of A. Pliś [14] that every solution of class $C^{1}$ is generated by a family of characteristic strips. Using this result, we can prove the following

Lemma 4.4. 1) Suppose that $f(x, y, z, p, q)=0$ is of Hamilton-Jacobi type. Then the boundary value problem

$$
\begin{cases}f(x, y, z, \partial z / \partial x, \partial z / \partial y)=0 & \text { in } D_{+} \\ (z, \partial z / \partial x, \partial z / \partial y)=\left(z_{\Gamma}, p_{\Gamma}, q_{\Gamma}\right) & \text { on } \Gamma\end{cases}
$$

has two solutions in a neighbourhood of the point $A$, and these two solutions coincide with the solutions constructed as above by the characteristic method.

2) Suppose that $f(x, y, z, p, q)=0$ is of Hamilton-Jacobi type. Then the boundary value problem

$$
\begin{cases}f(x, y, z, \partial z / \partial x, \partial z / \partial y)=0 & \text { in } D_{-} \\ (z, \partial z / \partial x, \partial z / \partial y)=\left(z_{\Gamma}, p_{\Gamma}, q_{\Gamma}\right) & \text { on } \Gamma\end{cases}
$$

has no solution in a neighbourhood of the point $A$.

In the quasi-linear case also, we can obtain a similar result.

Lemma 4.5. 1) Suppose that $f(x, y, z, p, q)=0$ is quasi-linear. Then the boundary value problem

$$
\begin{cases}f(x, y, z, \partial z / \partial x, \partial z / \partial y)=0 & \text { in } D_{+}, \\ z=z_{\Gamma} & \text { on } \Gamma,\end{cases}
$$

has two solutions in a neighbourhood of the point $A$, and these two solutions coincide with the solutions constructed as above by the characteristic method.

2) Suppose that $f(x, y, z, p, q)=0$ is quasi-linear. Then the boundary value problem

$$
\begin{cases}f(x, y, z, \partial z / \partial x, \partial z / \partial y)=0 & \text { in } D_{-}, \\ z=z_{\Gamma} & \text { on } \Gamma,\end{cases}
$$

has no solution in a neighbourhood of the point $A$.

Summing up Lemmas 4.4 and 4.5, we get the following 
Theorem 4.6. 1) Assume the conditions (A.1), (A.2), (A.3) and (A.4). Moreover, assume that the equation (4.2) is of Hamilton-Jacobi type. Then the extension of solution of the Cauchy problem (2.1)-(4.1) is unique in the space of $C^{1}$-functions which are of class $C^{2}$ except on piecewise smooth curves.

2) Assume the conditions (A.1), (A.2), (A.3) and (A.4). Moreover, assume that the equation (4.2) is quasi-linear. Then the extension of solution of the Cauchy problem (2.1)(4.1) is unique in the space of $C^{0}$-functions which are of class $C^{1}$ except on piecewise smooth curves.

R e m a rk. We will explain the meaning of this theorem, using the terminology given in Def. 4.1. Let $S$ be a solution surface whose Legendrian lift is constructed by a family of characteristic strips which are solutions of (4.3)-(4.4). This construction of solution is possible even if $S$ may contain singularities. Theorem 4.6 means that there are no other solutions which have the same regularity property as $S$.

5. Examples. The following two examples suggest that one can produce various kinds of first order partial differential equations as "intermediate integrals".

\section{EXAMPLE 1.}

$$
\left\{\begin{array}{l}
r t-s^{2}=0 \\
z(0, y)=\varphi(y), \quad p(0, y)=\varphi^{\prime}(y)^{2}, \quad q(0, y)=\varphi^{\prime}(y)
\end{array}\right.
$$

where $\varphi(y) \in C_{0}^{\infty}\left(\mathbb{R}^{1}\right)$. The first integrals of $r t-s^{2}=0$ are $\{p, q, z-p x-q y\}$. Therefore the intermediate inetgral for (5.1) is given by $f=p-q^{2}$. Hence the solution of (5.1) is obtained as a solution of the following Cauchy problem (5.2) for the Hamilton-Jacobi equation:

$$
\partial z / \partial x-(\partial z / \partial y)^{2}=0, \quad z(0, y)=\varphi(y)
$$

EXAMPLE 2.

$$
\left\{\begin{array}{l}
q^{2} r-p q s+z\left(r t-s^{2}\right)=0 \\
z(0, y)=\varphi(y), \quad p(0, y)=\varphi(y) \varphi^{\prime}(y), \quad q(0, y)=\varphi^{\prime}(y),
\end{array}\right.
$$

where $\varphi(y) \in C_{0}^{\infty}\left(\mathbb{R}^{1}\right)$. The first integrals of this equation are $\{p, z q\}$, and the intermediate integral is $f=p-z q$. Therefore a solution for (5.3) is given by a solution of the following conservation law:

$$
\partial z / \partial x-z(\partial z / \partial y)=0, \quad z(0, y)=\varphi(y)
$$

\section{References}

[1] R. Courant and D. Hilbert, Methods of Mathematical Physics, Vol. 2, Interscience, New York, 1962.

[2] G. Darboux, Leçons sur la théorie générale des surfaces, tome 3, Gauthier-Villars, Paris, 1894.

[3] E. Goursat, Leçons sur l'intégration des équations aux dérivées partielles du second ordre, tome 1, Hermann, Paris, 1932.

[4] —, Cours d'analyse mathématique, tome 3, Gauthier-Villars, Paris, 1927. 
[5] J. Hadamard, Le problème de Cauchy et les équations aux dérivées partielles linéaires hyperboliques, Hermann, Paris, 1932.

[6] S. Izumiya, Geometric singularities for Hamilton-Jacobi equation, in: Adv. Stud. Pure Math. 22, 1993, 89-100.

[7] S. Izumiya and G. T. Kossioris, Semi-local classification of geometric singularities for Hamilton-Jacobi equations, preprint.

[8] H. Lewy, Über das Anfangswertproblem bei einer hyperbolischen nichtlinearen partiellen Differentialgleichung zweiter Ordnung mit zwei unabhängigen Veränderlichen, Math. Ann. 98 (1928), 179-191.

[9] -, A priori limitations for solutions of Monge-Ampère equations I, II, Trans. Amer. Math. Soc. 37 (1934), 417-434; 41 (1937), 365-374.

[10] V. V. Lychagin, Contact geometry and non-linear second order differential equations, Russian Math. Surveys 34 (1979), 149-180.

[11] T. Morimoto, La géométrie des équations de Monge-Ampère, C. R. Acad. Sci. Paris 289 (1979), 25-28.

[12] S. Nakane, Formation of singularities for Hamilton-Jacobi equations in several space variables, J. Math. Soc. Japan 43 (1991), 89-100.

[13] —, Formation of shocks for a single conservation law, SIAM J. Math. Anal. 19 (1988), 1391-1408.

[14] A. Pliś, Caharacteristic of nonlinear partial differential equations, Bull. Acad. Polon. Sci. Cl. III 2 (1954), 419-422.

[15] M. Tsuji, Formation of singularities for Hamilton-Jacobi equation II, J. Math. Kyoto Univ. 26 (1986), 299-308.

[16] - Prolongation of classical solutions and singularities of generalized solutions, Ann. Inst. H. Poincaré Anal. Non Linéaire 7 (1990), 505-523.

[17] H. Whitney, On singularities of mappings of Euclidean spaces I, Ann. of Math. 62 (1955), 374-410. 